\title{
The parameters to estimate postoperative severe complications classified through Clavien-Dindo after upper abdominal surgery in patients with primary and recurrent ovarian cancer
}

\author{
Yakup Yalcin ${ }^{1,2}$, Burak Tatar ${ }^{3}$, Ebru Erdemoglü ${ }^{4}$, Evrim Erdemoglu ${ }^{5}$ \\ ${ }^{1}$ Department of Obstetrics and Gynecology, Istinye University School of Medicine, Istanbul, Turkey \\ ${ }^{2}$ Department of Gynecologic Oncology, Medical Park Hospital, Antalya, Turkey \\ ${ }^{3}$ Department of Gynecologic Oncology, Samsun Training and Research Hospital, Samsun, Turkey \\ ${ }^{4}$ Department of Obstetrics and Gynecology, Isparta State Hospital, Isparta, Turkey \\ ${ }^{5}$ Department of Gynecologic Oncology, Suleyman Demirel University, Isparta, Turkey
}

\begin{abstract}
Objectives: The more surgical effort and performing extensive upper abdominal surgery (UAS) are often required to accomplish the highest rates of optimally cytoreduction in patients with ovarian cancer. Nonetheless, the rate of complications increases with extensive surgery. We have studied the upper abdominal surgery complications by Clavien-Dindo Classification (CDC) and analyzed parameters affecting post-operative severe complications classified through Clavien-Dindo.

Material and methods: A retrospective cohort of patients diagnosed with epithelial ovarian cancer from January 1 st 2009 to April 30th 2016 was evaluated. Patients who underwent at least one UAS procedure with or without optimal cytoreduction for epithelial ovarian cancer (stage IIIC-IV or recurrent) were included. Postoperative complications were recorded according to the Clavien-Dindo Classification.

Results: In total, 58 patients were included. There were 120 UAS procedures performed on the 58 patients. Diaphragm peritonectomy was the most performed surgery $(50 \%, 29 / 58)$, and then the other UAS procedures were liver surgery $(39.7 \%$, $23 / 58)$, cholecystectomy $(24.1 \%, 14 / 58)$, splenic surgery $(24.1 \%, 14 / 58)$, full-thickness diaphragm resection $(22.4 \%, 13 / 58)$, pancreatic surgery $(19 \%, 11 / 58)$, resection of tumor from porta hepatis $(17.2 \%, 10 / 58)$, celiac lymph node excision $(8.6 \%$, $5 / 58)$, partial gastrectomy $(1.7 \%, 1 / 58)$, respectively. Thirteen patients $(22.4 \%)$ had post-operative grade $3-5$ complications according to $C D C$ within 30 days after surgery.

Conclusions: This current study demonstrated that the addition of extensive upper abdominal surgery procedures were not associated with increased postoperative severe complications in patients with recurrent or advanced ovarian cancer. These procedures are safe and feasible for patients in need and also can be performed with acceptable mortality and morbidity. Key words: upper abdominal surgery; Clavien-Dindo; ovarian cancer; postoperative complication
\end{abstract}

Ginekologia Polska 2019; 90, 10: 557-564

\section{INTRODUCTION}

Ovarian cancer is the second most common malignancy of reproductive tract in women and epithelial ovarian cancer (EOC) is the most fatal gynecologic cancer; the number of new cases of ovarian cancer is 11.9 per 100,000 whereas the number of deaths is 7.5 per 100,000 [1]. Despite the devastating survival statistics compared to other gynecologic cancers, there has been a decline in the mortality rate of EOC. Death rates have been falling on average $2.2 \%$ each year between 2004-2013 and the 5-year survival rate has an upward trend, increasing from $33.7 \%$ in 1977 to $46.2 \%$ in 2008 , although the majority of ovarian cancer patients have metastasis to upper abdominal organs at diagnosis and found to have stage III or stage IV disease [1]. Progress in life expectancy of ovarian cancer patients can mainly be attributed to advances in cytoreductive surgery and implementation of platin based chemotherapy $[2,3]$. 
Major developments in medical treatment of EOC are: introduction and combination of paclitaxel to platin based chemotherapy in 1996 [4]; the emergence of intraperitoneal chemotherapy in 2006 [5]; and targeted chemotherapies and poly [adenosine diphosphate (ADP)] ribose polymerase (PARP) inhibitors recently [6]. The evolution of surgical treatment of EOC was towards more radical, extensive procedures that consisted of radical oopherectomy, pelvic peritonectomy, bowel resections and anastomosis, and then upper abdominal procedures were added to armory of gynecologic oncologists [7]. The concept of optimal cytoreduction was changing and brought to an end at maximal cytoreduction in order to improve overall survival, despite the new medical treatment modalities [8]. There has been afierce debate over extensive maximal debulking surgery versus neoadjuvant treatment. However, many studies have shown that primary cytoreductive surgery (CRS) aiming no residual disease was the most important modifiable prognostic factor affecting survival [9-11]. A dedicated gynecologic oncology team performing extensive upper abdominal surgery (UAS) such as diaphragm stripping and/or resection, liver resection, cholecystectomy, splenectomy, distal pancreatectomy, resection of tumor from porta hepatis, celiac lymph node excision and partial gastrectomy is required to accomplish the highest rates of optimally cytoreduction or complete resection [11-13]. The comprehension of CRS and the biology of a tumor was established by many reports on the impact of CRS and upper abdominal surgery on oncological outcomes. However, systematic evaluation of complications of upper abdominal surgery is seldom studied. Additionally, standardization is required for classification of surgical complications. There is no consensus among gynecologic oncologist on how to report surgical complications.

Clavien-Dindo Classification (CDC), mainly used by general surgeons, has been proposed to rank a complication in an objective, reliable and reproducible manner [14]. The point of CDC is mainly on the therapeutic consequences of a complication. Therefore, we have studied the upper abdominal surgery complications by CDC and analyzed parameters affecting post-operative severe complications classified through Clavien-Dindo.

\section{MATERIAL AND METHODS}

We obtained Institutional Review Board approval (number: 04/03/15:55) and then identified all patients with International Federation of Gynecology and Obstetrics (FIGO) stage IIIC and IV epithelial ovarian cancer or recurrent epithelial ovarian cancer who underwent extensive upper abdominal surgery at the Suleyman Demirel University Hospital from January 1, 2009 thru April 30, 2016. The medical records of all patients were retrospectively reviewed for the following data: age, body mass index (BMI) American Society of Anesthesiologist (ASA) score, Eastern Cooperative Oncology Group (ECOG) performance status, FIGO stage, pre-operative albumin, serum cancer antigen (CA125), hemoglobin levels, ascites, upper abdominal surgery procedures, estimated blood loss, intraoperative blood transfusion, duration of surgery, residual disease after surgery, length of hospital stay, and finally post-operative complications within 30 days and pathologic data.

We included patients who had undergone at least one upper abdominal surgery procedure with or without optimal cytoreduction for epithelial ovarian cancer (FIGO stages IIIC-IV or recurrent). We excluded patients who had received neoadjuvant chemotherapy or histologically confirmed non-epithelial ovarian cancers, low malignant potential tumors from the study.

These patients were classified according to residual disease (RD); RD 0: no residual disease, $R D$ 1-10: residual disease 1-10 mm, and RD > 10: gross residual disease is more than $10 \mathrm{~mm}$. Extensive surgical procedures were performed on the upper abdomen included diaphragm peritonectomy, full-thickness diaphragm resection, liver surgery (partial liver resection or segmental hepatectomy or liver capsule metastasectomy), cholecystectomy, splenic surgery (splenectomy or resection of tumor on the surface of spleen without splenectomy), pancreatic surgery (distal pancreatectomy or resection of tumor on the pancreatic capsule), partial gastric resection, celiac lymph node excision, and resection of tumor from porta hepatis.

We recorded post-operative complications with the Clavien-Dindo Classification. We accepted post-operative complications or death associated with surgery if occuring within 30 days after surgery. Complications were evaluated in five categories depending on their severity in the CDC (1: no treatment or simple medical treatment, and 5: death) (Tab. 1). We subdivided patients into two groups; grade $1-2$ complications as mild and grade $3-5$ as a severe group. We focused on those grades with serious clinical outcomes. If patients had more than one complication, we noted the highest grade complication in the analysis. Adjuvant chemotherapy was routinely administered within 6 weeks of the operation.

Mean, standard deviation, median lowest, highest, frequency and ratio values were used in the descriptive statistics of the data. The distribution of the variables was measured by the Kolmogorov Simirnov Test. Mann-Whitney $U$ Test and Independent Sample T Test were used in the analysis of quantitative data. The Chi-square test was used to analyze qualitative data, and the Fisher test was used when the chi-square test conditions were not met. SPSS 22.0 program was used in the analyzes. 
Table 1. Clavien-Dindo Classification of surgical complications

\begin{tabular}{|c|c|}
\hline Grade & Definition \\
\hline Grade I & $\begin{array}{l}\text { Any deviation from the normal course without the need } \\
\text { for pharmacological treatment or surgical, endoscopic and } \\
\text { radiologic interventions. Allowed therapeutic regimens } \\
\text { are: drugs as antiemetics, antipyretics, analgetics, diuretics, } \\
\text { electrolytes and physiotherapy. This grade also includes } \\
\text { wound infections opened at the bedside }\end{array}$ \\
\hline Grade II & $\begin{array}{l}\text { Requiring pharmacological treatment with drugs other } \\
\text { than such allowed for grade I complications. Blood } \\
\text { transfusions and total parenteral nutrition are also included }\end{array}$ \\
\hline Grade III & Requiring surgical, endoscopic or radiological intervention \\
\hline IIIA & Intervention not under general anesthesia \\
\hline IIIB & Intervention under general anesthesia \\
\hline Grade IV & $\begin{array}{l}\text { Life-threatening complication (including CNS } \\
\text { complications)* requiring IC/ICU management }\end{array}$ \\
\hline IVA & Single organ dysfunction (including dialysis) \\
\hline IVB & Multiorgan dysfunction \\
\hline Grade V & Death of a patient \\
\hline
\end{tabular}

*Brain hemorrhage, ischemic stroke, subarachnoid bleeding, but excluding transient ischemic attacks; CNS — central nervous system; IC — intermediate care; ICU — intensive care unit

\section{RESULTS}

Fifty-eight patients with EOC who underwent upper abdominal surgery at our institution were included in this study between January 1, 2009 and April 30, 2016. All patients underwent cytoreductive surgery by exploratory laparotomy. The demographic characteristics and surgical outcomes were abstracted in Table 2.

The mean age was 62.2 , mean BMI was $27.6 \mathrm{~kg} / \mathrm{m}^{2}$, mean ascites volume was $919.3 \mathrm{ml}$, mean preoperative serum hemoglobin level was $12.4 \mathrm{~g} / \mathrm{dL}$, mean preoperative serum albumin level was $3.6 \mathrm{~g} / \mathrm{dL}$, mean preoperative serum CA125 was $799 \mathrm{u} / \mathrm{mL}$, mean estimated blood loss was $387.1 \mathrm{~mL}$, mean operative time was 319.1 minutes and mean post-operative hospital stay was 13.4 days. An intra-operative blood transfusion was required in 33 patients (56.8\%).

The most common ECOG performance status score was $0(36.2 \%)$ and $65.5 \%$ of patients had an ASA class of 2 . The majority of patients had serous histology ( $87.9 \%)$ and grade 3 tumors (67.3\%).

Thirty-two out of 58 patients (55.2\%) had primary disease and 26 patients (44.8\%) had recurrent disease. According to the results of cytoreductive surgery, no gross residual disease after surgery was in $58.6 \%$ ( $n: 34 / 58$, RD 0 ), gross residual disease $\leq 10 \mathrm{~mm}$ in $12.1 \%$ ( $\mathrm{n}: 7 / 58, \mathrm{RD}$ $1-10 \mathrm{~mm})$, and gross residual disease $>10 \mathrm{~mm}$ in $29.3 \%$ (n: $17 / 58, \mathrm{RD}>10 \mathrm{~mm}$ ). In patients who underwent debulking surgery was primary cytoreduction in $55.2 \%$ (32/58), secondary cytoreduction in $31.1 \%$ (18/58), tertiary cytoreduction in $13.7 \%$ (8/58) of cases respectively. Twenty-seven

\begin{tabular}{|c|c|c|}
\hline & Min-max & Mean + SD/N-\% \\
\hline Age [years] & $42.0-92.0$ & $62.2+10.6$ \\
\hline BMI $\left[\mathrm{kg} / \mathrm{m}^{2}\right]$ & $18.0-34.4$ & $27.6+4.1$ \\
\hline $\begin{array}{l}\text { Preoperative serum hemoglobin } \\
{[\mathrm{g} / \mathrm{dL}]}\end{array}$ & $9.2-15.4$ & $12.4+1.4$ \\
\hline $\begin{array}{l}\text { Preoperative serum albumin } \\
{[\mathrm{g} / \mathrm{dL}]}\end{array}$ & $1.6-4.8$ & $3.6+0.6$ \\
\hline Preoperative serum CA125 [u/mL] & $11.0-5005.0$ & $799.0+1140.1$ \\
\hline Ascites volume $[\mathrm{mL}]$ & $0-7000.0$ & $919.3+1490.1$ \\
\hline Operative time [min] & $140.0-570.0$ & $319.1+116.9$ \\
\hline Length of hospitalization [days] & $5.0-42.0$ & $13.4+8.2$ \\
\hline Estimated blood loss [mL] & $100-1700.0$ & $387.1+332.7$ \\
\hline $\begin{array}{l}\text { Intra-operative units of blood } \\
\text { transfused }\end{array}$ & & 3356.8 \\
\hline $\begin{array}{l}\text { ECOG performance status } \\
0 \\
1 \\
2 \\
3\end{array}$ & & $\begin{array}{l}2136.2 \\
1627.6 \\
1220.7 \\
915.5\end{array}$ \\
\hline $\begin{array}{l}\text { ASA score } \\
1 \\
2 \\
3\end{array}$ & & $\begin{array}{l}35.2 \\
3865.5 \\
1729.3\end{array}$ \\
\hline $\begin{array}{l}\text { Cytoreduction } \\
\text { Primary } \\
\text { Secondary } \\
\text { Tertiary }\end{array}$ & & $\begin{array}{l}3255.2 \\
1831.1 \\
813.7\end{array}$ \\
\hline $\begin{array}{l}\text { FIGO stage } \\
\text { IIIC } \\
\text { IV } \\
\text { Recurrent disease }\end{array}$ & & $\begin{array}{l}2746.6 \\
58.6 \\
2644.8\end{array}$ \\
\hline $\begin{array}{l}\text { Residual disease } \\
\qquad \begin{array}{l}\text { RD } 0 \\
\text { RD } 1-10 \mathrm{~mm} \\
\text { RD }>10 \mathrm{~mm}\end{array}\end{array}$ & & $\begin{array}{l}3458.6 \\
712.1 \\
1729.3\end{array}$ \\
\hline $\begin{array}{l}\text { Histology } \\
\text { Serous } \\
\text { Endometrioid } \\
\text { Mucinous } \\
\text { Carcinosarcoma } \\
\text { Transitional }\end{array}$ & & $\begin{array}{l}5187.9 \\
35.2 \\
1.7 \\
3.5 \\
11.7\end{array}$ \\
\hline $\begin{array}{l}\text { Tumor grade } \\
\qquad \begin{array}{l}1 \\
2 \\
3\end{array}\end{array}$ & & $\begin{array}{l}712.1 \\
1220.6 \\
3967.3\end{array}$ \\
\hline
\end{tabular}

PO - postoperative; BMI — body mass index; ASA — American Society of Anesthesiologist; ECOG — Eastern Cooperative Oncology Group; FIGO - International Federation of Gynecology and Obstetrics; CA125 - cancer antigen; RD — residual disease; SD — standard deviation

out of 32 patients (84.3\%) who had primary disease was stage IIIC and 5 patients (15.7\%) were stage VI according to FIGO classification.

There were 120 UAS procedures performed on the 58 patients, and multiple procedures were performed in many of patients. Diaphragm peritonectomy was the most 
performed surgery $(50 \%, 29 / 58)$, and then the other UAS procedures were liver surgery $(39.7 \%, 23 / 58)$, (right posterior bisegmentectomy (segment 6-7); 1/58, intraparenchymal tumor resections; $10 / 58$, liver capsule metastasectomy; $12 / 58)$, cholecystectomy $(24.1 \%, 14 / 58)$, splenic surgery $(24.1 \%, 14 / 58)$ (splenectomy; $12 / 58$, resection of the tumor on the surface of spleen; $2 / 58)$, full-thickness diaphragm resection $(22.4 \%, 13 / 58)$, pancreatic surgery $(19 \%, 11 / 58)$ (distal pancreatectomy; 4/58, resection of tumor on the pancreatic capsule; $7 / 58)$, resection of tumor from porta hepatis $(17.2 \%, 10 / 58)$, celiac lymph node excision $(8.6 \%, 5 / 58)$, partial gastrectomy $(1.7 \%, 1 / 58)$, respectively. Other surgical procedures implemented to patients were hysterectomy, unilateral/bilateral salpingo-oophorectomy, pelvic lymph node dissection, para-aortic lymph node dissection, omentectomy, peritonectomy, small bowel resection, large bowel resection, ileostomy, appendectomy, cardiophrenic lymph node dissection, VATS (video assisted thoracoscopic surgery), IP (intraperitoneal) catheter, and HIPEC (hyperthermic intraperitoneal chemotherapy) (Tab. 3).

Thirteen patients (22.4\%) had post-operative grade 3-5 complications according to Clavien-Dindo Classification within 30 days after surgery (Tab. 4). Ten patients (17.2\%) were reported as grade 3 complication, 1 patient (1.7\%) was reported as grade 4 complication, and 2 patients (3.5\%) were reported as grade 5 complication. Grade 3 complications were treated surgical, endoscopic or radiological intervention. Grade 4 complication was a life-threatening complication and treated at intensive care unit.

Two patients had grade 5 complications (mortalities) within 30 days of surgery $(3,5 \%)$. The first patient died due to short bowel syndrome in the $28^{\text {th }}$ post-operative day; she was a 49-year-old patient with an ECOG performance status of 2 who underwent multiple surgical procedures (right diaphragm peritonectomy, splenectomy, distal pancreatectomy, total colon resection, resection of the small intestine segment after the $70^{\text {th }} \mathrm{cm}$ of the treitz ligament, jejunostomy, HIPEC and RD 0 tertiary cytoreduction) for recurrent serous ovarian carcinoma. The second patient died due to acute cardiopulmonary failure in the $5^{\text {th }}$ post-operative day; she was an 87-year-old patient with an ECOG performance status of 3 and cardiac failure who underwent multiple surgical procedures (total abdominal hysterectomy with bilateral salpingo-oophorectomy, splenectomy, cholecystectomy, distal pancreatectomy, porta hepatis disease resection, total colectomy, ileostomy and optimal cytoreduction) for a stage 4 serous ovarian carcinoma. The patient's status was stable until the third postoperative day. On the third postoperative day, respiratory arrest occurred suddenly. She was taken to the intensive care unit and was connected to a ventilator
Table 3. Data of surgical procedures implemented to patients

Patients ( $N$ : 58)

n \%

\begin{tabular}{|l|l|}
\hline Upper abdominal surgery procedures (n: 120) & \\
\hline Diaphragm peritonectomy & $29 / 5850$ \\
\hline Full-thickness diaphragm resection & $13 / 5822.4$ \\
\hline Splenic surgery & $14 / 5824.1$ \\
\hline Pancreatic surgery & $11 / 5819.0$ \\
\hline Cholecystectomy & $14 / 5824.1$ \\
\hline Partial gastrectomy & $1 / 581.7$ \\
\hline Liver Surgery & $23 / 5839.7$ \\
\hline Celiac lymph node resection & $5 / 588.6$ \\
\hline Porta Hepatis disease resection & $10 / 5817.2$ \\
\hline Other procedures & \\
\hline Hysterectomy & $34 / 5858.6$ \\
\hline Unilateral/bilateral salpingo-oophorectomy & $34 / 5858.6$ \\
\hline Pelvic lymph node dissection & $25 / 5843.1$ \\
\hline Para-aortic lymph node dissection & $24 / 5841.3$ \\
\hline Omentectomy & $39 / 5867.2$ \\
\hline Peritonectomy & $29 / 5850$ \\
\hline Small bowel resection & $13 / 5822.4$ \\
\hline Large bowel resection & $21 / 5836.2$ \\
\hline Ileostomy & $2 / 583.4$ \\
\hline Colostomy & $4 / 586.9$ \\
\hline Anastomosis & $19 / 5832.7$ \\
\hline Appendectomy & $14 / 5824.1$ \\
\hline Cardiophrenic lymph node dissection & $1 / 581.7$ \\
\hline VATS & $7 / 5812.1$ \\
\hline IP catheter & $5 / 588.6$ \\
\hline HIPEC & $5 / 588.6$ \\
\hline VAS - video assistethor & \\
\hline
\end{tabular}

VATS - video assisted thoracoscopic surgery; IP — intraperitoneal; HIPEC - hyperthermic intraperitoneal chemotherapy

Table 4. Post-operative grade 3-5 complications according to Clavien-Dindo Classification

\begin{tabular}{|l|l|}
\hline Type of complication & $\begin{array}{l}\text { UAS Patients } \\
\text { (N: 58\%) }\end{array}$ \\
\hline Anastomotic insufficiency & $2 / 583.5$ \\
\hline Short bowel syndrome* & $1 / 581.7$ \\
\hline Pleural effusion & $1 / 581.7$ \\
\hline Pneumothorax & $1 / 581.7$ \\
\hline Pulmonary embolism & $1 / 581.7$ \\
\hline Postop bleeding & $1 / 581.7$ \\
\hline Acute cardiopulmonary failure* & $1 / 581.7$ \\
\hline Intra-abdominal abscess & $1 / 581.7$ \\
\hline Wound infection & $2 / 583.5$ \\
\hline Evisceration & $2 / 583.5$ \\
\hline Total complication & $\mathbf{1 3 / 5 8 2 2 . 4}$ \\
\hline
\end{tabular}

*Postoperative 30-day mortality (3.5\%); UAS - Upper Abdominal Surgery 
device and monitored. Her status worsened and she died on postoperative day 5 .

We analyzed the parameters for prediction of the major postoperative complication and mortality after extensive upper abdominal surgery. There was no statistical difference in age, BMI, ascites, preoperative serum hemoglobin level, preoperative serum albumin level, preoperative serum CA125 level, estimated blood loss, operative time, ECOG performance status score, ASA score, FIGO stage and residual disease between complicated and uncomplicated patients ( $p>0.05$ ). Only the length of the post-operative hospital stay was statistically significant between complicated and uncomplicated patients $(p<0.05)$ (Tab. 5).

No statistical difference was found between the length of the post-operative hospital stay and the types of upper abdominal surgical procedures ( $p>0.05$ ) (Tab. 6).

There was also no statistical difference in upper abdominal surgery procedures between complicated and uncomplicated patients ( $p>0.05$ ). Only diaphragm peritonectomy was statistically significant $(p<0.05)$ (Tab. 7).

\section{DISCUSSION}

The amount of residual tumors after surgery in patients with advanced stage cancer is closely related to disease free and overall survival [11]. Ovarian cancer tends to spread to the upper abdominal anatomic sites and organs and therefore upper abdominal surgery has a key role to achieve the optimally cytoreduction rate [15]. There are very few studies to indicate the complication rate in patients with extensive upper abdominal surgeries. Kuhn et al. [12] reported that the rate of perioperative serious complication increased in patients with advanced ovarian cancer who underwent UAS compared to standard surgery for tumor debulking. Chi et al. demonstrated that the rate of postoperative major complications in patients underwent extensive UAS was $22 \%$ and the rate of postoperative mortality was $1.4 \%$. This postoperative mortality and morbidity rate was acceptable [16]. In a population-based systematic review an average postoperative mortality after primary cytoreductive surgery for advanced stage epithelial ovarian cancer was reported as $2.5-3.7 \%$ [17]. In our study, the mortality rate was $3.4 \%$ and was similar to the literature.

Table 5. Comparison of parameters between patients with or without severe complications

\begin{tabular}{|c|c|c|c|c|c|c|}
\hline & & \multicolumn{2}{|c|}{$\begin{array}{l}\text { PO severe complication } \\
\text { no }\end{array}$} & \multicolumn{2}{|c|}{$\begin{array}{l}\text { PO severe complication } \\
\text { yes }\end{array}$} & \multirow{2}{*}{$\mathbf{p}$} \\
\hline & & Mean + SD/n-\% & Min-Max & Mean + SD/n-\% & Min-Max & \\
\hline \multicolumn{2}{|l|}{ Age [years] } & $61.5 \pm 10.2$ & $42-92$ & $64.5 \pm 12.1$ & $45-87$ & 0.355 \\
\hline \multicolumn{2}{|l|}{ BMI $\left[\mathrm{kg} / \mathrm{m}^{2}\right]$} & $27.7 \pm 3.8$ & $18-34$ & $27.2 \pm 5.0$ & $21-34$ & 0.532 \\
\hline \multicolumn{2}{|c|}{ Preoperative serum hemoglobin $[\mathrm{g} / \mathrm{dL}]$} & $12.4 \pm 1.3$ & $10-15$ & $12.4 \pm 1.8$ & $9-15$ & 0.881 \\
\hline \multicolumn{2}{|c|}{ Preoperative serum albumin [g/dL] } & $3.7 \pm 0.7$ & $1.6-4.8$ & $3.5 \pm 0.5$ & $2.6-4.2$ & 0.170 \\
\hline \multicolumn{2}{|c|}{ Preoperative serum CA125 [u/mL] } & $827.8 \pm 1190.5$ & $11-5005$ & $699.2 \pm 981.4$ & $22-3372$ & 0.758 \\
\hline \multicolumn{2}{|l|}{ Ascites volume $[\mathrm{mL}]$} & $815 \pm 1454$ & $0-7000$ & $1281 \pm 1617$ & $0-4000$ & 0.665 \\
\hline \multicolumn{2}{|l|}{ Operative time [min.] } & $313.8 \pm 118.4$ & $140-570$ & $337.7 \pm 114.4$ & $180-560$ & 0.520 \\
\hline \multicolumn{2}{|c|}{ Length of hospitalization [days] } & $11.0 \pm 4.4$ & $5-24$ & $21.9 \pm 12.0$ & $5-42$ & 0.001 \\
\hline \multicolumn{2}{|l|}{ Estimated blood loss [mL] } & $353.3 \pm 282.7$ & $100-1300$ & $503.8 \pm 462.1$ & $100-1700$ & 0.285 \\
\hline \multirow{4}{*}{ ECOG performance status } & 0 & $1635.6 \%$ & & $538.5 \%$ & & \multirow{4}{*}{0.393} \\
\hline & 1 & $1431.1 \%$ & & $215.4 \%$ & & \\
\hline & 2 & $920.0 \%$ & & $323.1 \%$ & & \\
\hline & 3 & $613.3 \%$ & & $323.1 \%$ & & \\
\hline \multirow{3}{*}{ ASA score } & 1 & $36.7 \%$ & & $00.0 \%$ & & \multirow{3}{*}{0.896} \\
\hline & 2 & $2964.4 \%$ & & $969.2 \%$ & & \\
\hline & 3 & $1328.9 \%$ & & $430.8 \%$ & & \\
\hline \multirow{3}{*}{ FIGO Stage } & $3 C$ & $2248.9 \%$ & & $538.5 \%$ & & \multirow{3}{*}{0.600} \\
\hline & 4 & $24.4 \%$ & & $323.1 \%$ & & \\
\hline & Recurrent & $2146.7 \%$ & & $538.5 \%$ & & \\
\hline \multirow{3}{*}{ Residual Disease } & No Visible & $2760.0 \%$ & & $753.8 \%$ & & \multirow{3}{*}{0.210} \\
\hline & $1-10 \mathrm{~mm}$ & $36.7 \%$ & & $430.8 \%$ & & \\
\hline & $>10 \mathrm{~mm}$ & $1533.3 \%$ & & $215.4 \%$ & & \\
\hline
\end{tabular}

T-test, Mann-Whitney U test, Chi-square test; PO — postoperative; BMI — body mass index; ASA — American Society of Anesthesiologist; ECOG — Eastern Cooperative Oncology Group; FIGO — International Federation of Gynecology and Obstetrics; CA125 — cancer antigen; SD — standard deviation 
Table 6. Comparison of length of hospital stay and types of surgical procedures

\begin{tabular}{|c|c|c|c|c|}
\hline & \multicolumn{4}{|c|}{ Length of Hospital Stay } \\
\hline & Min-Max & Median & Mean + SD & p \\
\hline $\begin{array}{l}\text { Diaphragm Resection No } \\
\text { Yes }\end{array}$ & $\begin{array}{l}5.0-42.0 \\
8.0-33.0\end{array}$ & $\begin{array}{l}11 \\
12\end{array}$ & $\begin{array}{l}13.2+8.4 \\
14.2+7.5\end{array}$ & 0.437 \\
\hline $\begin{array}{l}\text { Diaphragm Peritonectomy No } \\
\text { Yes }\end{array}$ & $\begin{array}{l}5.0-24.0 \\
5.0-42.0\end{array}$ & $\begin{array}{l}10 \\
12\end{array}$ & $\begin{array}{l}10.8+4.2 \\
16.1+10.2\end{array}$ & 0.057 \\
\hline $\begin{array}{l}\text { Splenic Surgery No } \\
\text { Yes }\end{array}$ & $\begin{array}{l}5.0-42.0 \\
5.0-33.0\end{array}$ & $\begin{array}{l}11 \\
11\end{array}$ & $\begin{array}{l}13.4+8.2 \\
13.7+8.2\end{array}$ & 0.827 \\
\hline $\begin{array}{l}\text { Cholecystectomy No } \\
\text { Yes }\end{array}$ & $\begin{array}{l}5.0-42.0 \\
5.0-24.0\end{array}$ & $\begin{array}{l}11 \\
11\end{array}$ & $\begin{array}{l}13.8+8.9 \\
12.2+5.1\end{array}$ & 0.956 \\
\hline $\begin{array}{l}\text { Gastric Surgery No } \\
\text { Yes }\end{array}$ & $\begin{array}{l}5.0-42.0 \\
28.0-28.0\end{array}$ & $\begin{array}{l}11 \\
28\end{array}$ & $\begin{array}{l}13.2+8.0 \\
28.0+-\end{array}$ & 0.142 \\
\hline $\begin{array}{l}\text { Pancreatic Surgery No } \\
\text { Yes }\end{array}$ & $\begin{array}{l}5.0-42.0 \\
5.0-33.0\end{array}$ & $\begin{array}{l}10 \\
13\end{array}$ & $\begin{array}{l}12.8+8.0 \\
16.1+8.9\end{array}$ & 0.164 \\
\hline $\begin{array}{l}\text { Liver Surgery No } \\
\text { Yes }\end{array}$ & $\begin{array}{l}5.0-38.0 \\
5.0-42.0\end{array}$ & $\begin{array}{l}11 \\
12\end{array}$ & $\begin{array}{l}12.7+7.0 \\
14.7+9.8\end{array}$ & 0.566 \\
\hline $\begin{array}{l}\text { Porta Hepatis Disease Resection No } \\
\text { Yes }\end{array}$ & $\begin{array}{l}5.0-42.0 \\
5.0-24.0\end{array}$ & $\begin{array}{l}11 \\
12\end{array}$ & $\begin{array}{l}13.6+8.6 \\
12.8+5.6\end{array}$ & 0.965 \\
\hline $\begin{array}{l}\text { Celiac Lymph Node Resection No } \\
\text { Yes }\end{array}$ & $\begin{array}{l}5.0-42.0 \\
8.0-18.0\end{array}$ & $\begin{array}{l}11 \\
11\end{array}$ & $\begin{array}{l}13.5+8.5 \\
12.4+4.4\end{array}$ & 0.813 \\
\hline
\end{tabular}

Mann-Whitney U test

Table 7. Comparison of postoperative severe complications and types of surgical procedures

\begin{tabular}{|l|l|l|l|}
\hline & $\begin{array}{l}\text { PO severe } \\
\text { complication } \\
\text { no }\end{array}$ & $\begin{array}{l}\text { PO severe } \\
\text { complication } \\
\text { yes }\end{array}$ & p \\
\cline { 1 - 3 } $\mathbf{n} \%$ & $\mathbf{n} \%$ & \\
\hline $\begin{array}{l}\text { Diaphragm Resection } \\
\text { Diaphragm }\end{array}$ & 1124.4 & 215.4 & 0.490 \\
\hline Peritonectomy & 1942.2 & 1076.9 & $\mathbf{0 . 0 2 8}$ \\
\hline Splenic Surgery & 920.0 & 538.5 & 0.171 \\
\hline Cholecystectomy & 1226.7 & 215.4 & 0.402 \\
\hline $\begin{array}{l}\text { Gastric Surgery } \\
\text { Pancreatic Surgery }\end{array}$ & $\mathbf{0 0 . 0}$ & 17.7 & 0.224 \\
\hline Liver Surgery & 1635.6 & 430.8 & 0.118 \\
\hline $\begin{array}{l}\text { Porta Hepatis Disease } \\
\text { Resection }\end{array}$ & 613.3 & 753.8 & 0.235 \\
\hline $\begin{array}{l}\text { Celiac Lymph Node } \\
\text { Resection }\end{array}$ & 48.9 & 17.7 & 0.143 \\
\hline PO pos. & & 1.000 \\
\hline
\end{tabular}

PO - postoperative, $x^{2}$ chi-square test (Fisher exact test)

In a previous study, the rate of postoperative major complications (grade 3-5) was reported as $19.8 \%$ in patients underwent UAS [18]. In our study, the rate of severe postoperative complications (grade $3-5$ ) was $22.4 \%$ and was compatible with this study.

In recent studies, liver surgery, splenectomy, pancreatic surgery, cholecystectomy, celiac lymphadenectomy and resection tumor from porta hepatis were reported as strong predictive factors for postoperative severe complications during cytoreductive surgery for advanced ovarian cancer [19-22]. We did not find any correlation between these procedures and postoperative severe complications. In our study, only diaphragm peritonectomy was associated with postoperative severe complications.

In the literature, the incidence of postoperative pleural effusion after diaphragmatic surgery as part of ovarian cancer debulking surgery ranged from $10 \%$ to $59 \%$ [23-26]. There is no consensus about use of a chest tube when the pleural space is opened during diaphragm surgery. Some authors do not recommend prophylactic use of a chest tube during diaphragm resection [18, 24-28], on the contrary, some authors routinely recommend chest tube placement [29-32]. Eisenhauer et al. [24] reported that the postoperative pleural effusion developed in $60 \%$ of the patients who underwent diaphragm surgery for advanced mullerian cancer and $15 \%$ of these patients required a postoperative chest tube placement or thoracentesis. In another study, the rate of postoperative pleural effusion following diaphragmatic peritonectomy with ovarian carcinoma was $30 \%$, and $12.5 \%$ of these patients were treated with thoracentesis or chest tube placement to manage symptomatic pleural effusions [25]. In these two studies, routine use of chest tubes were not recommended when the pleural space is opened. In contrast, Chereau and colleagues did not place a chest tube in patients whose pleural cavity was opened during diaphragm surgery with stage III/IV ovarian cancer (38\%) and the rate of postoperative chest tube placement was $27 \%$. Therefore, at the end of this study period, they decided to consist- 
ently place a chest tube [31]. Einenkel et al. [32] reported a high rate of postoperative chest tube placement (18\%) and recommended use of chest tubes during diaphragm resection. In our study, we routinely placed a chest tube during diaphragm resection (22.4\%) and necessity postoperative chest tube was $0 \%$ after diaphragm resection. We placed postoperative chest tubes because of symptomatic pleural effusion and pneumothorax in only 2 patients (3.4\%) whose were not performed diaphragm surgery.

Langstraat et al. [33] showed that low albumin level, emergent surgery, advanced age and stage IV disease were associated with poor surgical outcomes in multivariate analysis. Besides, they observed that increased surgical complexity did not increase the risk of postoperative major complications. In light of this information, extensive surgery should not be avoided in patients who require complex surgeries.

There are some scoring systems to predict postoperative complications. However, these scoring systems are neglected if you can completely remove the tumor in patients with advanced stage cancer [34]. Because, the maximal cytoreductive surgery is the most important prognostic factor for overall survival in patients with advanced ovarian cancer.

Ataseven et al. [35] reported that preoperative serum albumin level was a predictive factor for severe postoperative complications (grade 3-5). However, in another study preoperative serum, albumin levels were not associated with severe postoperative complications [16]. We didn't observed any significant relationship between serum albumin levels in patients with and without postoperative severe complications.

Chi et al. analyzed predictive factors for the risk of severe postoperative complications in patients underwent UAS. Parameters such as BMI, age, ASA score, FIGO stage, and preoperative CA-125 levels were found unrelated. However, ascites volume, estimated blood loss and operative time were reported as predictive factors [16]. In a recent study, BMI was reported as an independent risk factor for severe postoperative complications and mortality in patients underwent primary surgical debulking for ovarian cancer [35]. However, we did not find a correlation between these predictive factors and severe postoperative complications in our study.

Benedetti Panici et al. [18] showed that the types of surgical procedures (diaphragmatic, pancreatic, gastric resection and splenectomy) were significantly related to a longer postoperative stay. In our study, there was no correlation between the types of upper abdominal surgery procedures and the length of hospital stay. However, it was longer in patients with severe complication, this result may be due to longer treatment process.
In conclusion, this current study demonstrated that the addition of extensive upper abdominal surgery procedures were not associated with increased postoperative severe complications in patients with recurrent or advanced ovarian cancer. These procedures are safe and feasible for patients in need and also can be performed with acceptable mortality and morbidity.

\section{REFERENCES}

1. National Cancer Institute, Surveillance, Epidemiology, and End Results Program. https://seer.cancer.gov/statfacts/html/ovary.html (01.01.2017).

2. Griffiths $C T$. Surgical resection of tumor bulk in the primary treatment of ovarian carcinoma. Natl Cancer Inst Monogr. 1975; 42: 101-104, indexed in Pubmed: 1234624.

3. Hoskins WJ. Epithelial ovarian carcinoma: principles of primary surgery. Gynecol Oncol. 1994; 55(3 Pt 2): S91-S96, doi: 10.1006/gyno.1994.1346, indexed in Pubmed: 7835815.

4. McGuire WP, Hoskins WJ, Brady MF, et al. Cyclophosphamide and cisplatin compared with paclitaxel and cisplatin in patients with stage III and stage IV ovarian cancer. N Engl J Med. 1996; 334(1): 1-6, doi: 10.1056/NEJM199601043340101, indexed in Pubmed: 7494563.

5. Armstrong DK, Bundy B, Wenzel L, et al. Gynecologic Oncology Group. Intraperitoneal cisplatin and paclitaxel in ovarian cancer. N Engl J Med. 2006; 354(1): 34-43, doi: 10.1056/NEJMoa052985, indexed in Pubmed: 16394300.

6. Wiggans AJ, Cass GKS, Bryant A, et al. Poly(ADP-ribose) polymerase (PARP) inhibitors for the treatment of ovarian cancer. Cochrane Database Syst Rev. 2015(5): CD007929, doi: 10.1002/14651858.CD007929.pub3, indexed in Pubmed: 25991068.

7. Eisenkop SM, Friedman RL, Wang HJ. Complete cytoreductive surgery is feasible and maximizes survival in patients with advanced epithelial ovarian cancer: a prospective study. Gynecol Oncol. 1998;69(2): 103-108, doi: 10.1006/gyno.1998.4955, indexed in Pubmed: 9600815.

8. Chi DS, Eisenhauer EL, Lang J, et al. What is the optimal goal of primary cytoreductive surgery for bulky stage IIIC epithelial ovarian carcinoma (EOC)? Gynecol Oncol. 2006; 103(2): 559-564, doi: 10.1016/j. ygyno.2006.03.051, indexed in Pubmed: 16714056.

9. Winter WE, Maxwell GL, Tian C, et al. Gynecologic Oncology Group Study. Prognostic factors for stage III epithelial ovarian cancer: a Gynecologic Oncology Group Study. J Clin Oncol. 2007; 25(24): 3621-3627, doi: 10.1200/JCO.2006.10.2517, indexed in Pubmed: 17704411.

10. Fanfani F, Fagotti A, Ercoli A, et al. Is There a Role for Tertiary (TCR) and Quaternary (QCR) Cytoreduction in Recurrent Ovarian Cancer? Anticancer Res. 2015; 35(12): 6951-6955, indexed in Pubmed: 26637921.

11. Bristow RE, Tomacruz RS, Armstrong DK, et al. Survival effect of maximal cytoreductive surgery for advanced ovarian carcinoma during the platinum era: a meta-analysis. J Clin Oncol. 2002; 20(5): 1248-1259, doi: 10.1200/JCO.2002.20.5.1248, indexed in Pubmed: 11870167.

12. Kuhn W, Florack G, Roder J, et al. The influence of upper abdominal surgery on perioperative morbidity and mortality in patients with advanced ovarian cancer FIGO III and IV. Int J Gynecol Cancer. 1998; 8: 56-63.

13. Eisenkop SM, Spirtos NM, Friedman RL, et al. Relative influences of tumor volume before surgery and the cytoreductive outcome on survival for patients with advanced ovarian cancer: a prospective study. Gynecol Oncol. 2003; 90(2): 390-396, doi: 10.1016/s0090-8258(03)00278-6, indexed in Pubmed: 12893206.

14. Dindo D, Demartines N, Clavien PA. Classification of surgical complications: a new proposal with evaluation in a cohort of 6336 patients and results of a survey. Ann Surg. 2004; 240(2): 205-213, doi: 10.1097/01. sla.0000133083.54934.ae, indexed in Pubmed: 15273542.

15. Eisenhauer EL, Abu-Rustum NR, Sonoda Y, et al. The addition of extensive upper abdominal surgery to achieve optimal cytoreduction improves survival in patients with stages IIIC-IV epithelial ovarian cancer. Gynecol Oncol. 2006; 103(3): 1083-1090, doi: 10.1016/j.ygyno.2006.06.028, indexed in Pubmed: 16890277.

16. Chi DS, Zivanovic O, Levinson $\mathrm{KL}$, et al. The incidence of major complications after the performance of extensive upper abdominal surgical procedures during primary cytoreduction of advanced ovarian, tubal, and peritoneal carcinomas. Gynecol Oncol. 2010; 119(1): 38-42, doi: 10.1016/j.ygyno.2010.05.031, indexed in Pubmed: 20609464. 
17. Gerestein CG, Damhuis RAM, Burger CW, et al. Postoperative mortality after primary cytoreductive surgery for advanced stage epithelial ovarian cancer: a systematic review. Gynecol Oncol. 2009; 114(3): 523-527, doi: 10.1016/j.ygyno.2009.03.011, indexed in Pubmed: 19344936.

18. Benedetti Panici P, Di Donato V, Fischetti M, et al. Predictors of postoperative morbidity after cytoreduction for advanced ovarian cancer: Analysis and management of complications in upper abdominal surgery. Gynecol Oncol. 2015; 137(3):406-411, doi: 10.1016/j.ygyno.2015.03.043, indexed in Pubmed: 25824857.

19. Merideth MA, Cliby WA, Keeney GL, et al. Hepatic resection for metachronous metastases from ovarian carcinoma. Gynecol Oncol. 2003; 89(1): 1621, doi: 10.1016/s0090-8258(03)00004-0, indexed in Pubmed: 12694649.

20. Chen LM, Leuchter RS, Lagasse LD, et al. Splenectomy and surgical cytoreduction for ovarian cancer. Gynecol Oncol. 2000; 77(3): 362-368, doi: 10.1006/gyno.2000.5800, indexed in Pubmed: 10831343.

21. Kehoe SM, Eisenhauer EL, Abu-Rustum NR, et al. Incidence and management of pancreatic leaks after splenectomy with distal pancreatectomy performed during primary cytoreductive surgery for advanced ovarian, peritoneal and fallopian tube cancer. Gynecol Oncol. 2009; 112(3): 496500, doi: 10.1016/j.ygyno.2008.10.011, indexed in Pubmed: 19091388.

22. Magtibay PM, Adams PB, Silverman MB, et al. Splenectomy as part of cytoreductive surgery in ovarian cancer. Gynecol Oncol. 2006; 102(2): 369-374, doi: 10.1016/j.ygyno.2006.03.028, indexed in Pubmed: 16631919.

23. Gouy S, Chereau E, Custodio AS, et al. Surgical procedures and morbidities of diaphragmatic surgery in patients undergoing initial or interval debulking surgery for advanced-stage ovarian cancer. J Am Coll Surg. 2010; 210(4):509-514, doi: 10.1016/j.jamcollsurg.2010.01.011, indexed in Pubmed: 20347745.

24. Eisenhauer EL, D'Angelica MI, Abu-Rustum NR, et al. Incidence and management of pleural effusions after diaphragm peritonectomy or resection for advanced mullerian cancer. Gynecol Oncol. 2006; 103(3): 871-877, doi: 10.1016/j.ygyno.2006.05.023, indexed in Pubmed: 16815536.

25. Dowdy SC, Loewen RT, Aletti G, et al. Assessment of outcomes and morbidity following diaphragmatic peritonectomy for women with ovarian carcinoma. Gynecol Oncol. 2008; 109(2):303-307, doi: 10.1016/j. ygyno.2008.02.012, indexed in Pubmed: 18384866

26. Silver DF. Full-thickness diaphragmatic resection with simple and secure closure to accomplish complete cytoreductive surgery for patients with ovarian cancer. Gynecol Oncol. 2004; 95(2): 384-387, doi: 10.1016/j. ygyno.2004.07.046, indexed in Pubmed: 15491761.

27. Devolder K, Amant F, Neven $\mathrm{P}$, et al. Role of diaphragmatic surgery in 69 patients with ovarian carcinoma. Int J Gynecol Cancer. 2008; 18(2): 363-368, doi: 10.1111/j.1525-1438.2007.01006.x, indexed in Pubmed: 18334014.

28. Cliby W, Dowdy S, Feitoza SS, et al. Diaphragm resection for ovarian cancer: technique and short-term complications. Gynecol Oncol. 2004; 94(3): 655-660, doi: 10.1016/j.ygyno.2004.04.032, indexed in Pubmed: 15350355 .

29. Montz FJ, Schlaerth JB, Berek JS. Resection of diaphragmatic peritoneum and muscle: role in cytoreductive surgery for ovarian cancer. Gynecol Oncol. 1989; 35(3): 338-340, doi: 10.1016/0090-8258(89)90074-7, indexed in Pubmed: 2599468.

30. Kapnick SJ, Griffiths CT, Finkler NJ. Occult pleural involvement in stage III ovarian carcinoma: role of diaphragm resection. Gynecol Oncol. 1990; 39(2): 135-138, doi: 10.1016/0090-8258(90)90420-p, indexed in Pubmed: 2227587.

31. Chéreau $E$, Ballester $M$, Selle $F$, et al. Pulmonary morbidity of diaphragmatic surgery for stage III/IV ovarian cancer. BJOG. 2009; 116(8): 1062-1068, doi: 10.1111/j.1471-0528.2009.02214.x, indexed in Pubmed: 19459863.

32. Einenkel J, Ott R, Handzel R, et al. Characteristics and management of diaphragm involvement in patients with primary advanced-stage ovarian, fallopian tube, or peritoneal cancer. Int J Gynecol Cancer. 2009; 19(7): 1288-1297, doi: 10.1111/IGC.0b013e3181a3a833, indexed in Pubmed: 19823067.

33. Langstraat C, Aletti GD, Cliby WA. Morbidity, mortality and overal survival in elderly women undergoing primary surgical debulking for ovarian cancer: a delicate balance requiring individualization. Gynecol Oncol. 2011;123(2): 187-191, doi: 10.1016/j.ygyno.2011.06.031, indexed in Pubmed: 21794902

34. Chéreau E, Ballester M, Selle F. Comparison of peritoneal carcinomatosis scoring methods in predicting resectability and prognosis in advanced ovarian cancer. Am J Obstet Gynecol. 2010; 202: 178.e1-178.e10.

35. Ataseven B, du Bois A, Reinthaller A, et al. Pre-operative serum albumin is associated with post-operative complication rate and overall survival in patients with epithelial ovarian cancer undergoing cytoreductive surgery. Gynecol Oncol. 2015; 138(3): 560-565, doi: 10.1016/j. ygyno.2015.07.005, indexed in Pubmed: 26163893. 